\title{
Assessment of risk factors for postdural puncture headache in women undergoing cesarean delivery in Jordan: a retrospective analytical study
}

This article was published in the following Dove Press journal:

Local and Regional Anesthesia

17 March 2017

Number of times this article has been viewed

\author{
Wail N Khraise' \\ Mohammed Z Allouh ${ }^{2}$ \\ Khaled M El-Radaideh' \\ Raed S Said ${ }^{2}$ \\ Anas M Al-Rusan' \\ 'Department of Anesthesia, \\ ${ }^{2}$ Department of Anatomy, Faculty of \\ Medicine, Jordan University of Science \\ and Technology, Irbid, Jordan
}

Purpose: Postdural puncture headache (PDPH) is one of the most recognized complications after spinal anesthesia in women undergoing cesarean delivery. This study aimed to investigate the incidence of PDPH and its associated risk factors in women undergoing cesarean delivery in Jordan.

Patients and methods: This study included all women who underwent cesarean delivery at King Abdullah University Hospital in Jordan during 2015. Patient characteristics including age, weight, occurrence of PDPH, needle type, repeated puncture attempt, history of spinal anesthesia and PDPH, presence of tension headache, preeclampsia, migraine, sinusitis, and caffeine withdrawal were collated from hospital records. Statistical analyses were performed to assess the association of these characteristics with PDPH.

Results: The study cohort consisted of 680 women. Among these, only 43 (6.3\%) had developed PDPH. The only factors that showed significant association $(P<0.01)$ with PDPH were repeated puncture attempt and presence of tension headache. The repeated puncture attempt increased the risk of PDPH 2.55-fold, while presence of tension headache increased the risk 4.60-fold. Furthermore, the use of the traumatic $27 \mathrm{G}$ Spinostar needle increased the risk of repeated puncture attempt 28.45-fold $(P<0.01)$ compared with the use of the pencil-point $25 \mathrm{G}$ Whitacre needle. Conclusion: The major risk factors associated with the incidence of PDPH in women undergoing cesarean delivery in Jordan are repeated puncture attempt and presence of tension headache. The use of the pencil-point $25 \mathrm{G}$ Whitacre needle is recommended since this was associated with a substantially reduced risk of repeated spinal puncture than the traumatic 27 G Spinostar needle. Keywords: postdural puncture headache, postspinal puncture headache, spinal anesthesia, spinal needle

\section{Introduction}

Spinal anesthesia, also known as subarachnoid block, is a common type of regional anesthesia that involves the injection of an anesthetic agent into the subarachnoid space. ${ }^{1}$ It was first performed accidentally by Corning in $1885 .{ }^{2}$ Since then, it has been widely used, especially in obstetric patients undergoing cesarean delivery. However, despite its widespread use, the procedure is still associated with several complications; ${ }^{1}$ the most recognized is postdural puncture headache $(\mathrm{PDPH}){ }^{3}$

PDPH, also known as postspinal puncture headache, is an unpleasant complication that can develop after spinal anesthesia. Its incidence ranges between $<2 \%$ and $40 \%$ according to the needle type and size. ${ }^{4} \mathrm{PDPH}$ usually occurs within $1-2$ days after dural puncture, and the majority of patients respond to simple analgesia such as paracetamol, caffeine, bed rest, and good hydration maintenance. If the complication
Correspondence: Wail N Khraise Department of Anesthesia, Faculty of Medicine, Jordan University of Science and Technology, PO Box 3030, Irbid 22110, Jordan

Tel +962799051295

Fax +962 27201064

Email wnkhraise@just.edu.jo 
persists, an epidural blood patch should be administered. PDPH usually lasts between 5 and 7 days and is characterized by severe frontal or occipital headache that increases with sudden movement, getting up from supine position, coughing, and straining. In severe cases, there may be vision and hearing alterations as a result of traction on cranial nerves. ${ }^{5}$

Several risk factors have been attributed to $\mathrm{PDPH}$ including age, weight, needle size and design, and number of puncture attempts. ${ }^{5,6}$ For example, it has been reported that there is an inverse relationship between the incidence of PDPH and both age and weight. ${ }^{5}$ In addition, needle size and design appear to play a crucial role in the incidence of PDPH. ${ }^{4-6}$ Reducing the size of the spinal needle has been shown to significantly reduce the prevalence of PDPH. ${ }^{4,5}$

Until now, there has been no information about the incidence of PDPH in Jordanian women undergoing cesarean section. This study aimed to investigate the incidence of PDPH and its associated risk factors in women undergoing cesarean delivery in Jordan. It retrospectively analyzed the influence of several factors on the occurrence of PDPH. These factors included age, weight, needle type, repeated puncture attempt, previous history of spinal anesthesia, previous history of PDPH, presence of tension headache before anesthesia, preeclampsia, migraine, sinusitis, and caffeine withdrawal.

\section{Materials and methods}

The study cohort included all women who underwent cesarean delivery at King Abdullah University Hospital (KAUH) in Jordan during 2015. Women with a recorded history of chronic headache episodes of unknown cause were excluded from the study. This study was approved by the Institutional Research Board (IRB) committees at Jordan University of Science and Technology and KAUH (47/2016). Patients' consent to review their medical records was not required by the IRB committees. Patient data including age, weight, occurrence of spinal headache, needle type, repeated puncture attempt, previous history of spinal anesthesia and PDPH, presence of preeclampsia, migraine, and sinusitis were collated from the hospital records. In addition, the presence of tension headache before anesthesia and caffeine withdrawal were easily confirmed by questioning the patients as part of the original treatment and included in the patients' medical records.

The spinal anesthesia was performed by several anesthetists who have similar training and experience level using the same puncture technique and approach. The patients were followed up regularly for a period of 3 days. The follow-up assessments were conducted twice daily, once during the day time shift and once during the on-call night period. During these follow-ups, the patients were assessed by the assigned specialist or the senior resident available, and the follow-up findings were recorded in the patients' file. In addition, the patients were asked to report to the hospital any complaint of headache after being discharged home.

The headache was diagnosed as PDPH if it fulfilled the criteria of International Classification of Headache Disorders 3rd edition (beta version) (ICHD-III beta): (i) dural puncture has been performed; (ii) headache has developed within 5 days of the dural puncture; and (iii) headache is not better accounted for by another ICHD-III beta diagnosis. ${ }^{7}$

\section{Statistical analysis}

Patient characteristics were recorded as the mean \pm standard deviation for continuous variables and the frequency distribution for categorical variables. Chi-square test of association was applied to investigate the association between each variable and the occurrence of PDPH at the 5\% level of significance. In addition, binary logistic regression was performed to determine the adjusted relative risk of these variables on the incidence of PDPH.

\section{Results}

The study cohort comprised 680 women who underwent cesarean surgery under spinal anesthesia during 2015. Among these, 43 (6.3\%) women had developed PDPH. Approximately one-half of the studied population (335 women, 49.3\%) were anesthetized using a $25 \mathrm{~g}$ Whitacre needle type, while the others (345 women, 50.7\%) were anesthetized using a $27 \mathrm{G}$ Spinostar needle type. However, no significant association $(P>0.05)$ was found between the needle type and the incidence of PDPH. Furthermore, six women had a history of preeclampsia, while 19 women had a medical history of migraine. None of these patients had developed PDPH indicating that migraine and preeclampsia are not predisposing factors for PDPH in Jordanian women. The only two factors that showed a significant association $(P<0.01)$ with PDPH were repeated puncture attempt for a second time and presence of tension headache before spinal anesthesia. The frequency distribution of each factor with PDPH, along with its significance value, is shown in Table 1.

After controlling for the effects of all factors reported in Table 1, the logistic regression analysis revealed that patients who received a second puncture attempt had a 2.55-fold $(P<0.05)$ increased risk of developing PDPH, while patients who had tension headache prior to spinal anesthesia had a 4.60 -fold $(P<0.01)$ increased risk (Table 2$)$. The adjusted 
Table I Incidence of postdural puncture headache in relation to different factors

\begin{tabular}{|c|c|c|c|}
\hline Risk factors & No & Yes & $P$-value \\
\hline Age, mean \pm SD (years) & $31.5 \pm 0.22$ & $32.8 \pm 0.96$ & 0.120 \\
\hline Weight, mean \pm SD $(\mathrm{kg})$ & $83.1 \pm 0.4 I$ & $85.2 \pm 1.90$ & 0.275 \\
\hline \multicolumn{4}{|l|}{ Needle type, n (\%) } \\
\hline 25 G Whitacre & $318(94.9)$ & $17(5.1)$ & \multirow{2}{*}{0.187} \\
\hline 27 G Spinostar & $319(92.5)$ & $26(7.5)$ & \\
\hline \multicolumn{4}{|c|}{ Repeated puncture attempt, n (\%) } \\
\hline No & $448(95.3)$ & $22(4.7)$ & \multirow{2}{*}{0.008} \\
\hline Yes & $189(90.0)$ & $21(10.0)$ & \\
\hline \multicolumn{4}{|c|}{ Previous spinal anesthesia, $\mathrm{n}(\%)$} \\
\hline No & 481 (93.9) & $3 I(6.1)$ & \multirow{2}{*}{0.736} \\
\hline Yes & $151(93.2)$ & II (6.8) & \\
\hline \multicolumn{4}{|c|}{ Previous spinal headache, $\mathrm{n}(\%)$} \\
\hline No & $616(94.2)$ & $38(5.8)$ & \multirow{2}{*}{0.348} \\
\hline Yes & $16(88.9)$ & $2(I I . I)$ & \\
\hline \multicolumn{4}{|c|}{ Presence of tension headache, $\mathrm{n}(\%)$} \\
\hline No & $543(95.6)$ & $25(4.4)$ & \multirow{2}{*}{$<0.001$} \\
\hline Yes & $94(83.9)$ & $18(16.1)$ & \\
\hline \multicolumn{4}{|l|}{ Preeclampsia, n (\%) } \\
\hline No & $631(93.6)$ & $43(6.4)$ & \multirow{2}{*}{0.523} \\
\hline Yes & $6(100)$ & $0(0)$ & \\
\hline \multicolumn{4}{|l|}{ Migraine, n (\%) } \\
\hline No & $618(93.5)$ & $43(6.5)$ & \multirow{2}{*}{0.251} \\
\hline Yes & $19(100)$ & $0(0)$ & \\
\hline \multicolumn{4}{|l|}{ Sinusitis, n (\%) } \\
\hline No & $586(93.5)$ & $4 \mid(6.5)$ & \multirow{2}{*}{0.427} \\
\hline Yes & $51(96.2)$ & $2(3.8)$ & \\
\hline \multicolumn{4}{|l|}{ Caffeine withdrawal, n (\%) } \\
\hline No & 617 (93.9) & $40(6.1)$ & \multirow{2}{*}{0.178} \\
\hline Yes & $20(87.0)$ & $3(13.0)$ & \\
\hline
\end{tabular}

Abbreviations: $n$, number; $S D$, standard deviation.

Table 2 Adjusted effects of repeated puncture attempt and presence of headache on postdural puncture headache

\begin{tabular}{llll}
\hline Risk factor & P-value & AR & $\mathbf{9 5 \%} \mathbf{C l}$ \\
\hline Repeated puncture & & & \\
$\quad$ No & Ref & 1.00 (Ref) & Ref \\
Yes & 0.03 & 2.55 & $1.09-5.93$ \\
Presence of headache & & & \\
$\quad$ No & Ref & 1.00 (Ref) & Ref \\
Yes & $<0.001$ & 4.60 & $2.31-9.15$ \\
\hline
\end{tabular}

Notes: Factors in the logistic regression model included needle type, previous spina anesthesia, previous spinal headache, preeclampsia, migraine, caffeine withdrawal, sinusitis, age, and weight.

Abbreviations: AR, adjusted risk; $\mathrm{Cl}$, confidence interval; Ref, reference.

effects of the remaining factors were not significantly associated with the incidence of PDPH $(P>0.05)$. Furthermore, we tested if any of these factors were associated with repeated puncture attempt. The chi-square analysis revealed a significant $(P<0.01)$ association between needle type and repeated puncture attempt. The adjusted effects in the logistic regression model showed that the use of the 27 G Spinostar needle increased the risk of repeated puncture attempt 28.45 -fold compared with the use of the $25 \mathrm{G}$ Whitacre needle.

\section{Discussion}

To our knowledge, this is the first retrospective study to investigate the incidence of PDPH and its associated risk factors in women undergoing cesarean delivery in Jordan. The findings revealed that the most influential factors affecting PDPH incidence in these obstetrical patients were repeated puncture attempt and the presence of tension headache prior to the spinal anesthesia procedure. Furthermore, age, weight, previous history of spinal anesthesia and PDPH, presence of preeclampsia, migraine, sinusitis, and caffeine withdrawal did not predispose the women to PDPH. In addition, the study demonstrated that there was no direct effect of needle type on the incidence of PDPH. However, findings showed a clear correlation between needle type and repeated puncture attempt during spinal anesthesia.

Previous studies have reported contrary results to our findings regarding some of the aforementioned factors including the age of the patients, weight, and previous history of spinal anesthesia and PDPH. Seeberger et $\mathrm{al}^{8}$ reported an inverse correlation between the age of the patients and the incidence of PDPH. Moreover, Amorim and Valença ${ }^{9}$ demonstrated that patients with a history of previous PDPH have a significantly increased risk of developing a recurrent episode of PDPH. Faure et $\mathrm{al}^{10}$ found that morbidly obese pregnant women exhibited a lower incidence of PDPH due to increased intraabdominal pressure that leads to reduced cerebrospinal fluid (CSF) leakage. In this study, the insignificant role of these risk factors in the incidence of PDPH in Jordanian women suggests that the correlation between PDPH and these risk factors may vary based on racial and ethnic differences.

The pathophysiological mechanism of PDPH is still completely unclear. Puncturing the dura during spinal anesthesia may lead to excessive leakage of CSF, resulting in a marked decrease in intracranial pressure. ${ }^{4,11}$ This rapid rate of CSF loss cannot be immediately compensated by the slower rate of CSF production. ${ }^{12}$ It has been postulated that the reduction in intracranial pressure may cause a downward traction and stretching of the pain-sensitive intracranial structures in the upright position. These intracranial structures include cranial nerves, bridging veins, and the dura itself. ${ }^{4}$ The pain may be transferred along the trigeminal nerve to produce headache in the frontal head region and possibly also via the glossopharyngeal, vagus, and cervical spinal nerves to produce pain in the neck, shoulders, and occipital area of the head. ${ }^{13}$ Another speculated mechanism attributes PDPH to the vasodilation of meningeal vessels that occurs as a compensatory mechanism for the restoration of intracranial pressure to its normal value. ${ }^{14}$ The latter mechanism is consistent with the 
Monroe-Kellie doctrine which states that the total volume of the brain, blood, and CSF must always be constant within the cranial cavity; therefore, any decrease in the CSF volume must be compensated by an increase in the blood volume. ${ }^{14}$

Multiple studies had shown that needle characteristics, such as type and size, play a major role in the incidence of PDPH. ${ }^{15-18}$ Vallejo et al reported that the use of classical cutting bevel needles such as Quincke produces a higher frequency of PDPH compared with the more modern blunt pencil-point needles such as Whitacre. ${ }^{6}$ In addition, Vandam and Dripps reported a significant increase in PDPH incidence when $16 \mathrm{G}$ needles were used in comparison with $26 \mathrm{G}$ needles. ${ }^{19}$ In the present study however, there was no significant difference in the incidence of PDPH between the patients who were anesthetized with the beveled $27 \mathrm{G}$ Spinostar needle and those anesthetized with the pencilpoint $25 \mathrm{G}$ Whitacre needle. This lack of difference may be attributed to the smaller size of the traumatic Spinostar needle compared with the atraumatic Whitacre needle, indicating that it does not cause a more severe tear in the dura mater or a greater amount of CSF leakage compared with the Whitacre needle.

Other predisposing factors in the incidence of PDPH are puncture technique and needle orientation. ${ }^{20}$ Previous studies have showed that paramedian or lateral injections of anesthetic solution induce a smaller rate of CSF leakage compared with the median approach. ${ }^{21,22}$ The direction of the needle bevel also plays a major role in PDPH development. It has been shown that inserting the needle with its bevel parallel to the longitudinal fibers of the dura will cause a smaller puncture hole, resulting in a slower rate of CSF leakage. ${ }^{23}$ However, assessing the influence of these factors was not feasible in this study because all injections were performed by the same puncture technique and approach.

Our results showed that the repeated puncture attempt during spinal anesthesia is a major risk factor for PDPH. Repeated puncture attempts may lead to more perforations in the dura mater and thereby increase CSF loss. This correlation has also been reported by previous studies. ${ }^{8,24}$ Seeberger et $\mathrm{al}^{8}$ reported that $4.2 \%$ of patients who received a second puncture attempt developed PDPH compared to $1.6 \%$ of patients who required a single attempt. In addition, although needle type did not have a direct impact on the incidence of PDPH in this study, it was significantly correlated with repeated puncture attempt. Using the traumatic $27 \mathrm{G}$ Spinostar needle significantly increased the probability of repeating the spinal puncture for a second time compared with using the less traumatic $25 \mathrm{G}$ Whitacre needle. This therefore suggests that needle type is indirectly correlated with the incidence of PDPH in these obstetric patients. It is speculated that small needle sizes may be deviated or damaged by the calcified ligaments (i.e., ligamentum flavum) necessitating the repetition of the puncture. Indeed, an increase in the number of puncture attempts is typically observed with smaller spinal needles. ${ }^{25}$

The results of this study show that the presence of tension headache prior to spinal anesthesia may greatly increase the incidence of PDPH. An interrelationship between the mechanisms that generate PDPH and other types of headache has been suggested. ${ }^{26}$ Kuntz et $\mathrm{al}^{27}$ found that the group of patients with headache 1 week before the procedure had a much higher incidence of PDPH compared with PDPH incidence in non-headache group. Jabbari et al proposed that the unusual physiological status and the unclear nature of neurogenic transmitters in patients with chronic tension headache may render them more susceptible to developing subsequent episodes of PDPH. ${ }^{5}$

Regarding the management of PDPH, all patients were preloaded with $500 \mathrm{~mL}$ normal saline half an hour before the anesthetic procedure in order to reduce the risk of PDPH. However, patients who developed PDPH in this study were successfully managed by bed rest, increased fluid intake, and paracetamol prescription (1 g pro re nata). According to our hospital records, none of these patients required the administration of an epidural blood patch.

Some of the limitations to this study may include the sample size and the involvement of more than one anesthetist. However, it is important to note that the anesthetists who performed the spinal anesthesia in this study have similar training and experience level and they used the same puncture technique and approach. Nevertheless, further studies that include a larger sample and more influencing factors such as different puncture techniques are warranted.

\section{Conclusion}

Repeated puncture attempt and the presence of tension headache are major risk factors associated with the incidence of PDPH in women undergoing cesarean delivery in Jordan. Needle type did not have a direct impact on the incidence of the PDPH in these patients; however, it was directly related to repeated puncture attempt. It seems that the use of the pencilpoint $25 \mathrm{G}$ Whitacre needle is associated with a substantially reduced risk of repeated spinal puncture attempt than the use of the traumatic $27 \mathrm{G}$ Spinostar needle. Future investigations that involve a large sample with different needle types and puncture techniques are recommended. 


\section{Disclosure}

The authors report no conflicts of interest in this work.

\section{References}

1. Rodgers A, Walker N, Schug S, et al. Reduction of postoperative mortality and morbidity with epidural or spinal anaesthesia: results of overview of randomised trials. BMJ. 2000;321(7275):1493.

2. Corning JL. Spinal anesthesia and local medication of the cord. $N Y$ Med J. 1885;42:483-485.

3. Kuczkowski KM. Post-dural puncture headache in the obstetric patient: an old problem. New solutions. Minerva Anestesiol. 2004;70(12):823-830.

4. Turnbull DK, Shepherd DB. Post-dural puncture headache: pathogenesis, prevention and treatment. Br J Anaesth. 2003;91(5):718-729.

5. Jabbari A, Alijanpour E, Mir M, Bani Hashem N, Rabiea SM, Rupani MA. Post spinal puncture headache, an old problem and new concepts: review of articles about predisposing factors. Caspian J Intern Med. 2013;4(1):595-602.

6. Vallejo MC, Mandell GL, Sabo DP, Ramanathan S. Postdural puncture headache: a randomized comparison of five spinal needles in obstetric patients. Anesth Analg. 2000;91(4):916-920.

7. Hayter MA, Friedman Z, Katznelson R, Hanlon JG, Borges B, Naik VN. Effect of sleep deprivation on labour epidural catheter placement. Br J Anaesth. 2010;104(5):619-627.

8. Seeberger MD, Kaufmann M, Staender S, Schneider M, Scheidegger D. Repeated dural punctures increase the incidence of postdural puncture headache. Anesth Analg. 1996;82(2):302-305.

9. Amorim JA, Valença MM. Postdural puncture headache is a risk factor for new postdural puncture headache. Cephalalgia. 2008;28(1):5-8

10. Faure E, Moreno R, Thisted R. Incidence of postdural puncture headache in morbidly obese parturients. Reg Anesth. 1994;19(5):361-363.

11. Grant R, Condon B, Hart I, Teasdale GM. Changes in intracranial CSF volume after lumbar puncture and their relationship to post-LP headache. J Neurol Neurosurg Psychiatry. 1991;54(5):440-442.

12. Cruickshank RH, Hopkinson JM. Fluid flow through dural puncture sites. An in vitro comparison of needle point types. Anaesthesia. 1989; 44(5):415-418.

13. Horlocker TT. Complications of spinal and epidural anesthesia. Anesthesiol Clin North America. 2000;18(2):461-485.
14. Mokri B. The Monro-Kellie hypothesis: applications in CSF volume depletion. Neurology. 2001;56(12):1746-1748.

15. Lybecker H, Møller JT, May O, Nielsen HK. Incidence and prediction of postdural puncture headache: a prospective study of 1021 spinal anesthesias. Anesth Analg. 1990;70(4):389-394.

16. Halpern S, Preston R. Postdural puncture headache and spinal needle design. Metaanalyses. Anesthesiology. 1994;81(6):1376-1383.

17. Ross BK, Chadwick HS, Mancuso JJ, Benedetti C. Sprotte needle for obstetric anesthesia: decreased incidence of post dural puncture headache. Reg Anesth. 1992;17(1):29-33.

18. Tarkkila PJ, Heine H, Tervo RR. Comparison of Sprotte and Quincke needles with respect to post dural puncture headache and backache. Reg Anesth. 1992;17(5):283-287.

19. Vandam LD, Dripps RD. Long-term follow-up of patients who received 10,098 spinal anesthetics; syndrome of decreased intracranial pressure (headache and ocular and auditory difficulties). J Am Med Assoc. 1956; 161(7):586-591.

20. Janik R, Dick W. [Post spinal headache. Its incidence following the median and paramedian techniques]. Anaesthesist. 1992;41(3):137-141. German [with English abstract].

21. Angle PJ, Kronberg JE, Thompson DE, et al. Dural tissue trauma and cerebrospinal fluid leak after epidural needle puncture: effect of needle design, angle, and bevel orientation. Anesthesiology. 2003;99(6): 1376-1382.

22. Jones RJ. The role of recumbency in the prevention and treatment of postspinal headache. Anesth Analg. 1974;53(5):788-796.

23. Richman JM, Joe EM, Cohen SR, et al. Bevel direction and postdural puncture headache: a meta-analysis. Neurologist. 2006;12(4):224-228.

24. Hatfalvi BI. Postulated mechanisms for postdural puncture headache and review of laboratory models. Clinical experience. Reg Anesth. 1995; 20(4):329-336.

25. Shah VR, Bhosale GP. Spinal anaesthesia in young patients: evaluation of needle gauge and design on technical problems and postdural puncture headache. S Afr J Anaesthesiol Analg. 2010;16(3):24-28.

26. Oomura M, Yamawaki T, Miyashita K, Yamagami H, Naritomi H. Disappearance of migraine attacks during long-lasting postdural puncture headache: a case report. Headache. 2002;42(5):356-358.

27. Kuntz KM, Kokmen E, Stevens JC, Miller P, Offord KP, Ho MM. Postlumbar puncture headaches: experience in 501 consecutive procedures. Neurology. 1992;42(10):1884-1887.
Local and Regional Anesthesia

\section{Publish your work in this journal}

Local and Regional Anesthesia is an international, peer-reviewed open access journal publishing on the development, pharmacology, delivery and targeting and clinical use of local and regional anesthetics and analgesics. The journal is included in PubMed, and welcomes submitted papers covering original research, basic science, clinical studies,

\section{Dovepress}

reviews and evaluations, guidelines, expert opinion and commentary, case reports and extended reports. The manuscript management system is completely online and includes a very quick and fair peer-review system, which is all easy to use. Visit http://www.dovepress.com/ testimonials.php to read real quotes from published authors. 